\title{
Wnt5a-induced cell migration is associated with the aggressiveness of estrogen receptor-positive breast cancer
}

\author{
Yoshie Kobayashi', Takayuki Kadoya ${ }^{1}$, Ai Amioka ${ }^{1}$, Hideaki Hanaki ${ }^{1}$, Shinsuke \\ Sasada $^{1}$, Norio Masumoto ${ }^{1}$, Hideki Yamamoto ${ }^{2}$, Koji Arihiro $^{3}$, Akira Kikuchi ${ }^{2}$ and \\ Morihito Okada ${ }^{1}$ \\ ${ }^{1}$ Department of Surgical Oncology, Research Center for Radiation Casualty Medicine, Research Institute for Radiation Biology \\ and Medicine, Hiroshima University, Minami-ku, Hiroshima, 734-8551, Japan \\ ${ }^{2}$ Department of Molecular Biology and Biochemistry, Graduate School of Medicine, Osaka University, Suita, Osaka, 565- \\ 0871, Japan \\ ${ }^{3}$ Department of Anatomical Pathology, Hiroshima University Hospital, Minami-ku, Hiroshima, 734-8551, Japan \\ Correspondence to: Takayuki Kadoya, email: takayukikadoya@gmail.com \\ Keywords: Wnt5a; ER-positive breast cancer; cell migration; activated leukocyte cell adhesion molecule; prognosis \\ Received: August 25, $2017 \quad$ Accepted: February 26, $2018 \quad$ Published: April 20, 2018 \\ Copyright: Kobayashi et al. This is an open-access article distributed under the terms of the Creative Commons Attribution License \\ 3.0 (CC BY 3.0), which permits unrestricted use, distribution, and reproduction in any medium, provided the original author and \\ source are credited.
}

\section{ABSTRACT}

Elevated expression of Wnt5a is associated with malignancy, cell invasion, and metastasis. The role of Wnt5a expression in breast cancer remains elusive. We investigated the significance of Wnt5a expression in breast cancer. The relationship between Wnt5a expression and clinicopathologic factors was assessed in invasive breast cancer $(n=178)$ resected at Hiroshima University Hospital between January 2011 and February 2014. Wnt5a was expressed in 69 of 178 cases (39\%) of invasive breast cancer and correlated strongly with estrogen receptor (ER) expression $(P<0.001)$. Wnt5a expression in ER-positive breast cancer correlated significantly with lymph node metastasis, nuclear grade, and lymphatic invasion. The recurrencefree survival was shorter in breast cancer patients with Wnt5a expression than in those without $(P=\mathbf{0 . 0 2 4})$. The migratory capacity of ER-positive breast cancer cells increased with constitutive expression of Wnt5a and decreased with Wnt5a knockdown. DNA microarray analysis identified activated leukocyte cell adhesion molecule (ALCAM) as the primary gene induced by Wnt5a. ALCAM was expressed in $69 \%$ of Wnt5a-positive but only $27 \%$ of Wnt5a-negative cancers $(\kappa=0.444$; $P<0.001$ ). The inhibition of ALCAM reversed the enhanced migratory effect of Wnt5a, confirming the importance of this protein in the migration of ER-positive breast cancer cells. Wnt5a expression is related to high malignancy and a poor prognosis in ERpositive breast cancer. We suspect that Wnt5a expression increases the malignancy of breast cancer by increasing the migratory capacity of cancer cells through the induction of ALCAM expression.

\section{INTRODUCTION}

Wnt signaling occurs in $\beta$-catenin-dependent pathways, through $\beta$-catenin regulates the expression of many genes, and in the $\beta$-catenin-independent planar cell polarity and $\mathrm{Wnt} / \mathrm{Ca}^{2+}$ pathways $[1,2]$. $\beta$-catenindependent pathways are involved in some cancers, for example, familial polyposis, in which the adenomatous polyposis coli mutation causes the accumulation of $\beta$-catenin in the nucleus, resulting in colorectal cancer $[2,3]$. In $\beta$-catenin-independent pathways, Wnt5a is a representative activating ligand involved in cell motility and cell polarity through downstream signaling (e.g., JNK phosphorylation) [4]. Wnt5a expression correlates 
significantly with malignancy and stage of progression in malignant melanoma $[5,6]$, gastric cancer [7-9], prostate cancer [10], lung cancer [11], and pancreatic cancer [12]. In contrast, Wnt5a is a tumor suppressor in colorectal cancer [13], thyroid cancer [14], liver cancer [15], and malignant lymphoma [16]. Thus, the effect of Wnt5a expression differs between organs.

Whether Wnt5a expression in breast cancer correlates with pathological factors such as lymph node metastasis and grade is unknown. While a significant correlation between the immunohistochemical disappearance of Wnt5a expression and poor prognosis $[17,18]$ has been reported, these studies do not distinguish between breast cancers subtypes. Breast cancer is classified into four subtypes based on the expression of the estrogen receptor (ER) and human epidermal growth factor receptor 2 (HER2) [19, 20]. Because ER-positive and -negative breast cancers derive from different tissues (ductal epithelium and basal cells, respectively), the two subtypes are biologically distinct. ER-positive breast cancers progress slowly, whereas those that are ER-negative are highly malignant and have a poor prognosis. In previous studies, the inclusion of all subtypes, with their remarkably distinct prognoses, likely interfered with the accurate determination of the role of $\mathrm{Wnt} 5 \mathrm{a}$ in breast cancer.

A positive correlation is reported between $\mathrm{Wnt} 5 \mathrm{a}$ expression level and ER-positive breast cancer, as determined using breast cancer specimens [17, 18]. A correlation was observed between the PIK3CA mutation and Wnt5a expression based on the examination of 43 cases of ER-positive tumors [21]. Another study reports no significant correlation between the expression of Wnt5a and ER status, as determined by examination of 94 stained breast cancer specimens [22]. We believe that to determine the role of Wnt5a expression in breast cancer, the level of Wnt5a expression in each of the breast cancer subtypes must first be determined.

The mechanism of malignant transformation by Wnt5a has been studied in a variety of cancers. Cell motility in Wnt5a-positive gastric cancer is increased through activation of FAK and Rac to induce malignant transformation [7, 8]. One study of breast cancer reports an increase in expression of $\mathrm{Wnt} 5 \mathrm{a} / \mathrm{b}$ and their respective receptors Ror1 and 2 in brain metastases [23]. Other studies report that Wnt5a expression increases the malignancy of breast cancer through activation of tumor-related macrophages [24] or the promotion of cell migration [25]. However, to date, no study has described the mechanism underlying malignant transformation by Wnt5a in breast cancer.

In this study, we examine the significance of Wnt5a expression in breast cancer by determining the clinicopathologic characteristics of Wnt5a-positive breast cancers using Wnt5a immunohistochemical analysis of breast cancer specimens. We investigate the mechanism of malignant transformation in breast cancer by Wnt5a through biological analyses of cultured cells.

\section{RESULTS}

\section{Wnt5a is expressed in ER-positive breast cancer}

We observed weak Wnt5a expression in non-tumor ductal epithelial cells but none in basal or stromal cells (Figure 1Aa, 1Ab). Wnt5a was expressed in the cytoplasm but not the nucleus of breast cancer cells (Figure 1Aa, 1Ac). Wnt5a expression scores are shown in Figure 1B. Scores of $0,1+, 2+$, and $3+$ accounted for $20 \%, 25 \%, 16 \%$, and $39 \%$ of the specimen scores, respectively. A score of $3+$ was defined to be positive for Wnt5a expression.

Of the 178 cases of invasive breast cancer, 69 (39\%) were Wnt5a-positive and 109 (61\%) were Wnt5anegative (Table 1, Supplementary Table 1). A very strong correlation was observed between Wnt5a expression and positivity for ER or PgR. Wnt5a expression was extremely low in ER-negative breast cancers. There was no correlation between Wnt5a expression and HER2 positivity. Wnt5a-positive breast cancers were classified into subtypes based on ER status, as shown in Table 1.

\section{Wnt5a expression is associated with high-grade malignancy in ER-positive breast cancer}

Because we observed that Wnt5a expression is associated with ER-positive breast cancer, we investigated the pathological factors and prognosis of ER-positive breast cancers exclusively. Analysis of 153 ER-positive cases revealed a significant difference between Wnt5apositive and negative breast cancer in the presence of lymph node metastasis $(P<0.001)$, nuclear grade $(P=$ $0.004)$, and lymphatic invasion $(P=0.002)$ (Table 2$)$. Although no significant correlation was seen, there were clear trends toward a relationship between Wnt5a expression and the presence of vessel invasion $(P=$ $0.050)$, tumor size $(P=0.069)$, and Ki-67 labeling index $(P=0.058)$. These data suggest that malignancy is higher in Wnt5a-positive breast cancer that is also ER-positive rather than ER-negative. Comparison of recurrence-free survival, for which 5-year survival rates were $81.1 \%$ and $100 \%$ in Wnt5a-positive and Wnt5a-negative breast cancers, respectively, revealed a significant difference according to statistical analysis using the log-rank test ( $P$ $=0.024$ ) (Figure 2). The clinicopathological factors and recurrence sites of ER-positive breast cancer are shown in Supplementary Table 2.

\section{Migratory capacity of Wnt5a-expressing breast cancer cells}

We prepared MCF-7, an ER-positive breast cancer cell line expressing no Wnt5a, and forced it to express Wnt5a constitutively (MCF-7/Wnt5a cells). These cells showed no change in the levels of proteins involved in breast cancer-related signaling pathways or $\beta$-catenindependent pathways, such as ER, $\beta$-catenin, and cyclin 
D1 (Figure 3A). There was no significant difference in the proliferative capacity between MCF-7/Wnt5a and control MCF-7 cells (Figure 3B). As Wnt5a is reported to associate with cell migration, we conducted cell migration assays to determine the migratory capacity of Wnt5a- positive breast cancer cells. We also tested Wnt5a-silenced MCF-7/Wnt5a cells (MCF-7/Wnt5a + Wnt5a-siRNA cells), bringing the total number of cell types investigated to three. The migratory capacity of MCF-7/Wnt5a cells increased significantly $12 \mathrm{~h}$ later, whereas knockdown of

A

a

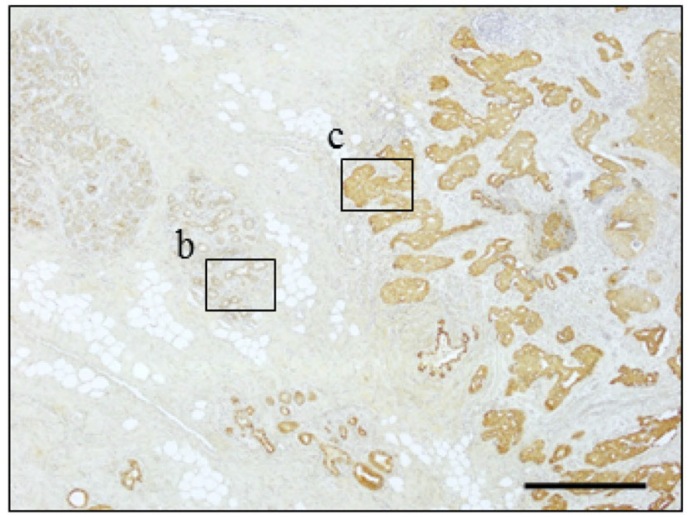

b Non-tumor mammary duct

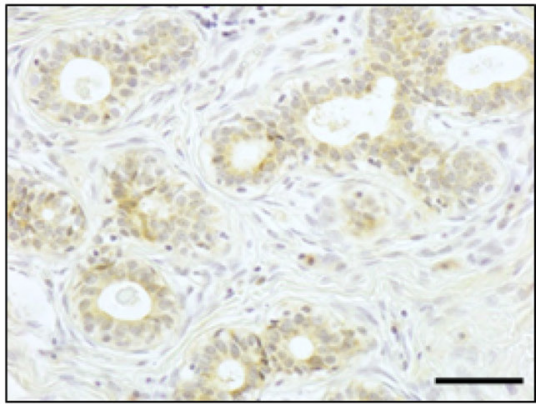

c Invasive breast cancer

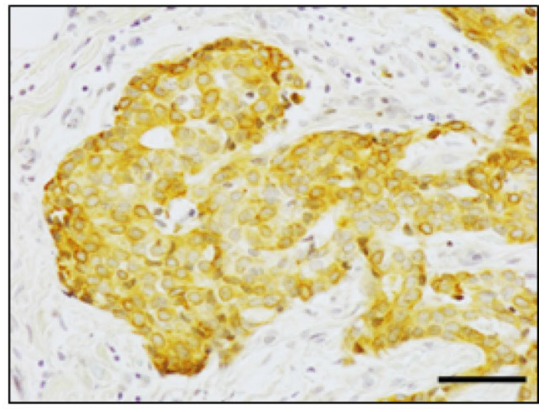

B
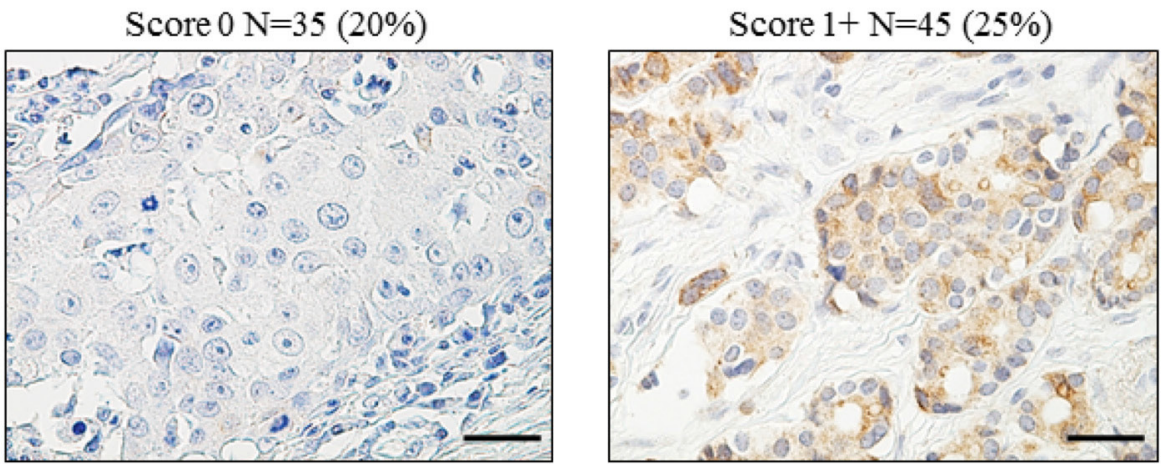

Score $2+\mathrm{N}=29(16 \%)$

Score $3+\mathrm{N}=69(39 \%)$
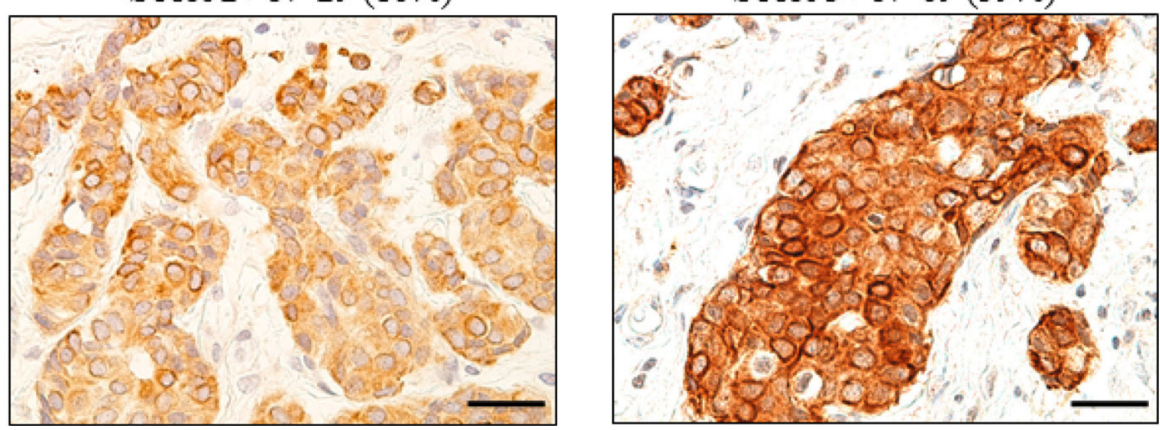

Figure 1: Wnt5a expression in breast cancer using breast cancer specimens. (A) Immunohistochemical staining for Wnt5a in invasive breast cancer. (a) Wnt5a expression in invasive breast cancer. (b) Wnt5a expressed weakly in cytoplasm in non-tumor mammary duct. (c) Wnt5a expressed strongly in invasive breast cancer a: bar, $500 \mu \mathrm{m}$, magnification $\times 40$; b, c: bar $50 \mu \mathrm{m}, \times 400$ (B) Evaluation of Wnt5a expression was scored as $0,1+, 2+$, or $3+$, taking into consideration both staining proportion and intensity. Scores $0-2+$, negative; Score $3+$, positive. bar, $50 \mu \mathrm{m}, \times 400$. 
Table 1: Relation of Wnt5a expression with ER, PgR and HER-2 in breast cancer

\begin{tabular}{lcccc}
\hline & & \multicolumn{3}{c}{ Wnt5a expression } \\
\cline { 3 - 4 } & Total $(\boldsymbol{n}=\mathbf{1 7 8})$ & Negative $(\boldsymbol{n}=\mathbf{1 0 9})$ & Positive $(\boldsymbol{n}=\mathbf{6 9})$ & $\boldsymbol{P}$ value \\
\hline ER, $N(\%)$ & $25(14)$ & $24(22)$ & $1(1)$ & \\
Negative & $153(86)$ & $85(78)$ & $68(99)$ & $<0.001$ \\
Positive & & & & \\
PgR, $N(\%)$ & $38(21)$ & $34(31)$ & $4(6)$ & $<0.001$ \\
Negative & $140(79)$ & $75(69)$ & $65(94)$ & \\
Positive & & & & \\
HER2, $N(\%)$ & $159(89)$ & $96(88)$ & $63(91)$ & 0.496 \\
Negative & $19(11)$ & $13(12)$ & & $6(9)$ \\
Positive & & &
\end{tabular}

Wnt5a resulted in a lower increase in migratory capacity (Figure 3C, 3D).

We investigated the endogenous Wnt5a expression in several ER-positive breast cancer cell lines. A strong endogenous Wnt5a expression was observed in MDAMB-175-VII cells (Figure 4A, Supplementary Figure 1). The knockdown of Wnt5a in MDA-MB-175-VII cells significantly decreased migratory capacity, whereas knockdown of Wnt5a in MDA-MB-361 cells did not decrease migratory capacity (Figure 4B).

\section{DNA microarray analysis using MCF-7/Wnt5a cells}

To investigate the mechanism by which Wnt5a expression leads to increased migratory activity, we conducted DNA microarray analysis of MCF-7/Wnt5a cells and control cells. We identified several genes upregulated (Table 3) and several downregulated (Supplementary Table 3) by Wnt5a. One of the upregulated genes, ALCAM, is a member of the immunoglobulin superfamily that is localized in the plasma membrane. ALCAM is involved in apoptosis, angiogenesis, migration, and invasion [26-28]. We focused on ALCAM and investigated its protein expression by Western blotting, observing a marked increase of ALCAM in MCF-7/ Wnt5a cells and decrease of ALCAM in MCF-7/Wnt5a and MDA-MB-175-VII cells by the knockdown of Wnt5a (Supplementary Figure 2, Figure 5A).

\section{ALCAM expression correlates with Wnt5a expression in ER-positive breast cancer}

We examined the expression of Wnt5a and ALCAM in breast cancer specimens. Scoring ALCAM expression as $0-3$, we observed that $46 \%$ had a score of $3+$, which was defined to be positive (Figure 5B). In 153 cases of ER-positive breast cancer, ALCAM was expressed in
$69 \%$ of Wnt5a-positive breast cancers but only $27 \%$ of Wnt5a-negative breast cancers, a statistically significant correlation between Wnt5a and ALCAM expression (Table 4) $(\kappa=0.444 ; P<0.001)$. Serial sections of pathological specimens of invasive breast cancer revealed that Wnt5a was expressed in the cytoplasm, whereas ALCAM was expressed in the plasma membrane (Figure 5C).

\section{Knockdown of ALCAM also reduces migratory capacity}

Our data indicate that the knockdown of Wnt5a reduced ALCAM expression and migratory capacity (Figures 3C, 3D and 4B). We next conducted cell migration assays to determine whether inhibition of ALCAM decreases migratory capacity as with the knockdown of Wnt5a. The migratory capacity of MDAMB-175-VII cells significantly decreased $12 \mathrm{~h}$ after the knockdown of ALCAM (Figure 6A, 6B), suggesting that cell migration is regulated via the Wnt5a-ALCAM signaling pathway in ER-positive breast cancer.

\section{DISCUSSION}

We examined nearly 200 specimens using an antibody already confirmed accurate in immunohistochemical analysis of Wnt5a-positive gastric cancers [7]. We observed a high correlation between Wnt5a and ER expression in breast cancers. In addition, our results indicate that Wnt5a-positivity strongly correlated with the presence of lymph node metastasis, lymphatic invasion, vessel invasion, and nuclear grade in ER-positive breast cancer. We also observed that among ER-positive breast cancers, those expressing Wnt5a were more malignant and had a poorer prognosis than those that did not express Wnt5a.

We also investigated the mechanism underlying malignant transformation by Wnt5a. Previous studies 


\begin{tabular}{|c|c|c|c|c|}
\hline & \multirow[b]{2}{*}{ Total $(N=153)$} & \multicolumn{3}{|c|}{ Wnt5a expression } \\
\hline & & Negative $(n=85)$ & Positive $(n=68)$ & $P$ value \\
\hline Age (median, range) & & $63,35-86$ & $57.5,34-87$ & 0.065 \\
\hline \multicolumn{5}{|l|}{ Age, $N(\%)$} \\
\hline$\leq 45$ & $28(18)$ & $13(15)$ & $15(22)$ & \\
\hline$>45$ & $125(82)$ & $72(85)$ & $53(78)$ & 0.282 \\
\hline \multicolumn{5}{|c|}{ Menopausal status, $N(\%)$} \\
\hline Premenopausal & $58(38)$ & $27(32)$ & $31(46)$ & \\
\hline Postmenopausal & $95(62)$ & $58(68)$ & $37(54)$ & 0.080 \\
\hline \multicolumn{5}{|l|}{ Tumor size, $N(\%)$} \\
\hline $\mathrm{pT} 1 \leq 20 \mathrm{~mm}$ & $104(44)$ & $63(74)$ & $41(60)$ & \\
\hline $\mathrm{pT} 2 / \mathrm{pT} 3>20 \mathrm{~mm}$ & $49(56)$ & $22(26)$ & $27(40)$ & 0.069 \\
\hline \multicolumn{5}{|c|}{ Lymph node metastasis, $N(\%)$} \\
\hline Negative & $103(67)$ & $72(85)$ & $31(46)$ & \\
\hline Positive & $50(33)$ & $13(15)$ & $37(54)$ & $<0.001$ \\
\hline \multicolumn{5}{|l|}{ Nuclear grade, $N(\%)$} \\
\hline $1 / 2$ & $85(56)$ & $56(66)$ & $29(43)$ & \\
\hline 3 & $68(44)$ & $29(34)$ & $39(57)$ & 0.004 \\
\hline \multicolumn{5}{|c|}{ Lymphatic invasion, $N(\%)$} \\
\hline Negative & $101(66)$ & $65(76)$ & $36(53)$ & \\
\hline Positive & $52(34)$ & $20(24)$ & $32(47)$ & 0.002 \\
\hline \multicolumn{5}{|l|}{ Vessel invasion, $N(\%)$} \\
\hline Negative & $142(93)$ & $82(96)$ & $60(88)$ & \\
\hline Positive & $11(7)$ & $3(4)$ & $8(12)$ & 0.050 \\
\hline \multicolumn{5}{|l|}{$\mathrm{Ki}-67, N(\%)$} \\
\hline $0-20$ & 67 (79) & $43(51)$ & $24(35)$ & \\
\hline $21-100$ & $86(56)$ & $42(49)$ & $44(65)$ & 0.058 \\
\hline
\end{tabular}

have reported that Wnt5a is involved in increased cell proliferation, differentiation, migration, adhesion, and changes in cell polarity [4]. We observed that the migratory capacity of ER-positive breast cancer cells forced to express Wnt5a increased and the knockdown of Wnt5a led to a decrease in migratory capacity, whereas cell proliferation remained unchanged. DNA microarray analysis revealed that ALCAM expression in Wnt5apositive breast cancer cells is induced by Wnt5a. ALCAM expression in breast cancer is reported to correlate with ER expression, lymph node metastasis, distant metastasis, and a poor prognosis $[26,30,31]$. Activation of the $\beta$-cateninindependent pathway induces ALCAM expression, and JNK located downstream of Wnt5a induces ALCAM expression [32]. We explored the function of Wnt5a and ALCAM on cell migration in ER-positive breast cancer and found that the knockdown of Wnt5a reduced ALCAM expression and cell migration. Furthermore, the knockdown of ALCAM also showed the reduction of cell migration, suggesting that cell migration is regulated via the Wnt5a-ALCAM signaling pathway in ER-positive breast cancer. Our investigation of breast cancer tissues also revealed that Wnt5a expression correlates with ALCAM expression in ER-positive breast cancers, indicating that Wnt5a-positive/ALCAM-positive breast cancers form a biologically distinct subgroup of ERpositive breast cancers.

Wnt5a expression has been shown to correlate with tumor progression in a variety of cancers. However, the function of Wnt5a has been reported as both a tumor 
suppressor and a tumor promotor in breast cancer. The loss of Wnt5a was found to be an indicator of poor prognosis in a study of whole breast cancers, including different subtypes [17]. However, accurate assessment of Wnt5a function in different subtypes is difficult because mutations in the signaling molecules differ between different subtypes. Therefore, it is important to determine which subtypes express Wnt5a. The majority of the previous reports on Wnt5a-positive breast cancer classified these cancers based solely on the presence or absence of Wnt5a expression, without regard to subtype. In addition, the number of cases studied in these reports was small, and there was not a suitable antibody available for immunohistochemical analysis of tissues.

Wnt5a overexpression was observed in ER-positive breast cancers with a mutation in PIK3CA [19]; PIK3CA mutations are seen in approximately $30 \%$ of ER-positive breast cancers and cause resistance to therapy [33, 34]. Together with these reports, our results suggest that Wnt5a is involved in the malignant transformation and/or relapse of ER-positive breast cancer. Wnt5a-Ror2 signals increase the expression of receptor activator of nuclear factor$\kappa \mathrm{B}$ (RANK) in osteoclast precursors by activating JNK and recruiting c-Jun, thereby enhancing RANK ligand (RANKL)-induced osteoclastogenesis [35]. Interestingly, all patients with recurrent breast cancer in this study had bone metastasis, suggesting that Wnt5a plays a role in bone metastasis in breast cancer.

Increased Wnt5a expression has been observed in ER-positive breast cancers with PIK3CA mutations [19]. Thus, we propose that mutations in $P I K 3 C A$ induce Wnt5a expression, which activates JNK to induce ALCAM expression, causing malignant transformation of ERpositive breast cancer cells. It is unknown whether Wnt5a

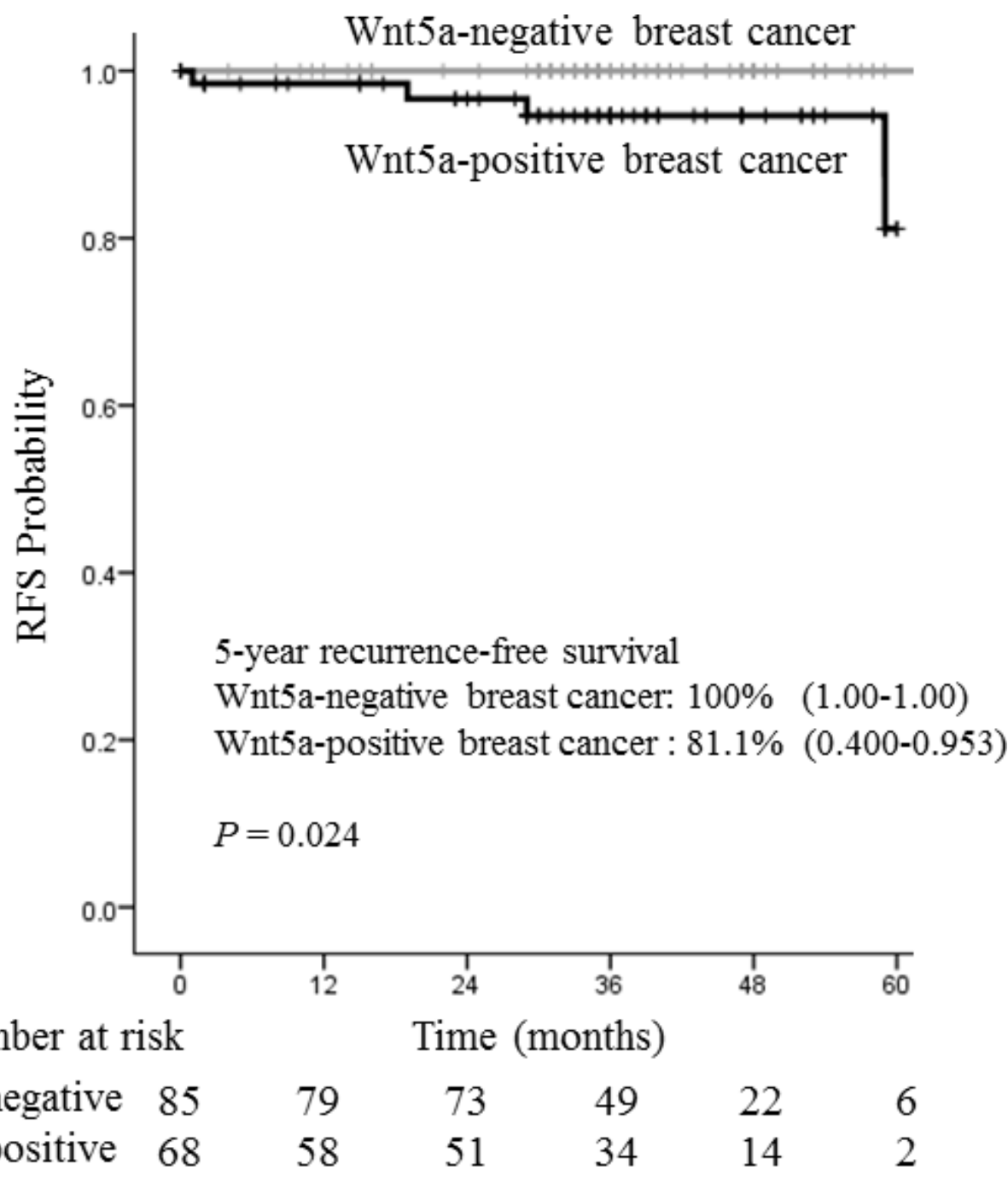

Figure 2: Relationship between recurrence-free survival and Wnt5a in 153 ER-positive breast cancer patients. The recurrence-free survival rates were analyzed according to Wnt5a immunohistochemistry. The mean 5-year recurrence-free survival rates were $100 \%$ for Wnt5a negative breast cancer and $81.1 \%$ for Wnt5a negative breast cancer. Wnt5a expression was significantly associated with poor recurrence-free survival in ER-positive cancer patients $(P=0.024)$. 
A

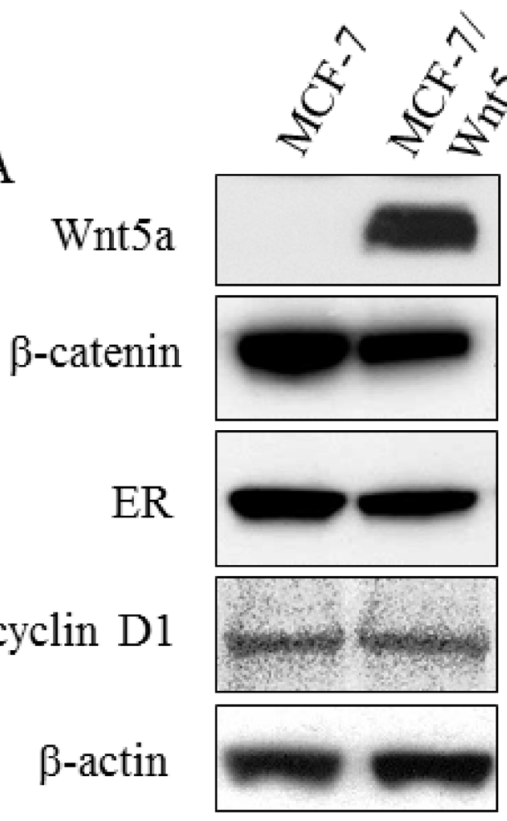

C

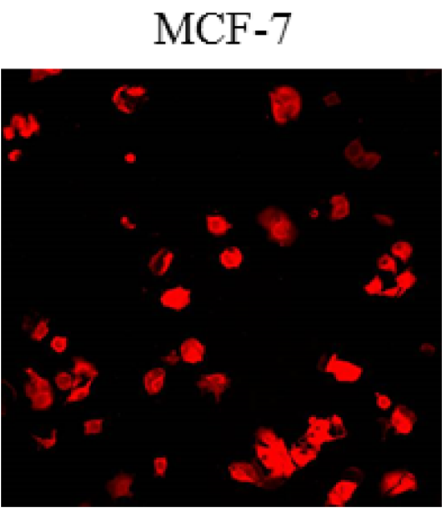

B

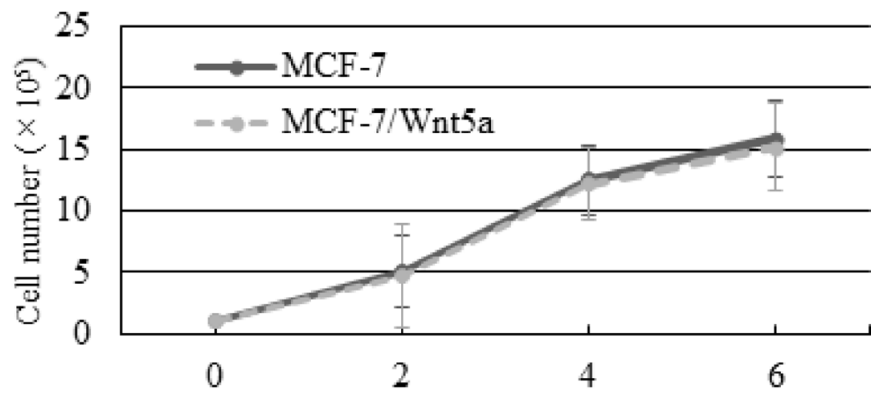

MCF-7/Wnt5a+sc
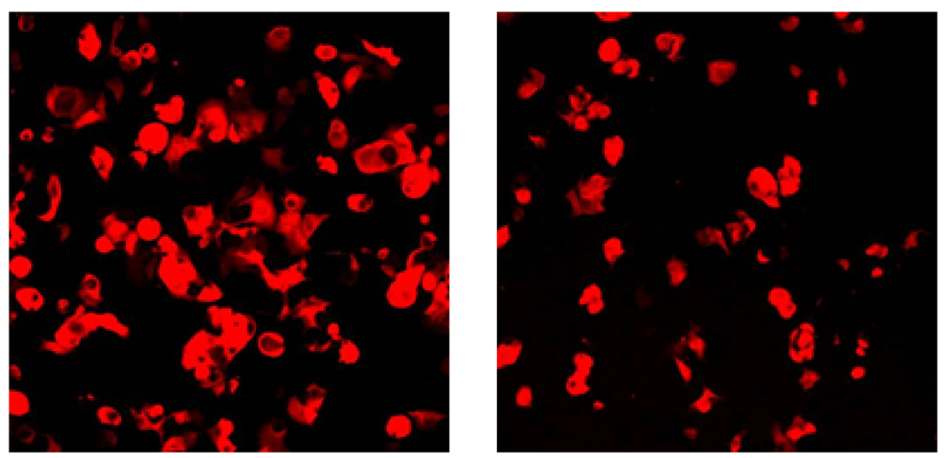

D

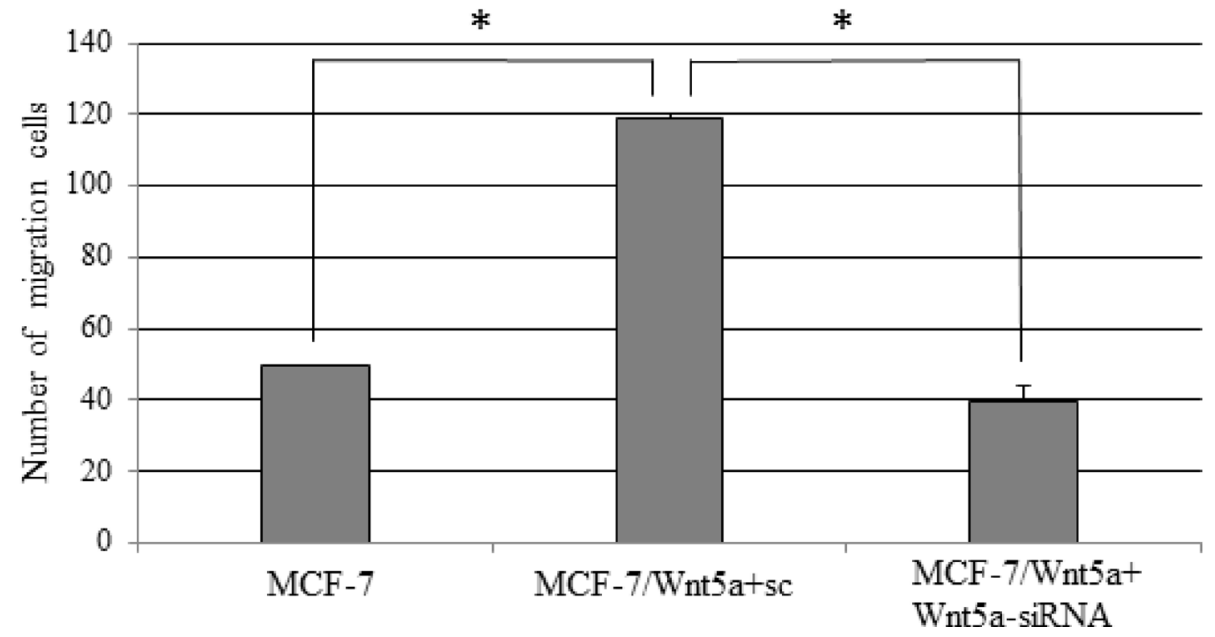

Figure 3: Migratory capacity of Wnt5a-expressing breast cancer cells. (A) The expression levels of $\beta$-catenin, ER, and cyclin D in MCF-7/Wnt5a and control MCF-7 cells. (B) The proliferative capacity in MCF-7/Wnt5a and control MCF-7 cells. Data are presented as the mean \pm SE of three proliferation assays. (C, D) The migratory capacity of MCF-7, MCF-7/Wnt5a + sc, and MCF-7/Wnt5a + Wnt5asiRNA cells. Data are presented as the mean \pm SE of three migration assays. Data were evaluated using the Mann-Whitney $U$-test. 
expression is induced by activation of $\mathrm{PI} 3 \mathrm{~K} / \mathrm{AKT} / \mathrm{mTOR}$ signaling caused by PIK3CA mutation in ER-positive breast cancers. This question will be the focus of our future studies.

In conclusion, Wnt5a induces malignant transformation in ER-positive breast cancers. Increased invasiveness resulting from upregulated ALCAM expression suggested the mechanism whereby Wnt5a induces malignant transformation. Wnt5a may be useful as a predictor of malignancy, a therapeutic target, and a prognostic indicator in ER-positive breast cancer.

\section{MATERIALS AND METHODS}

\section{Sample selection}

Patients with invasive breast cancer treated surgically between January 2011 and February 2014 at

A

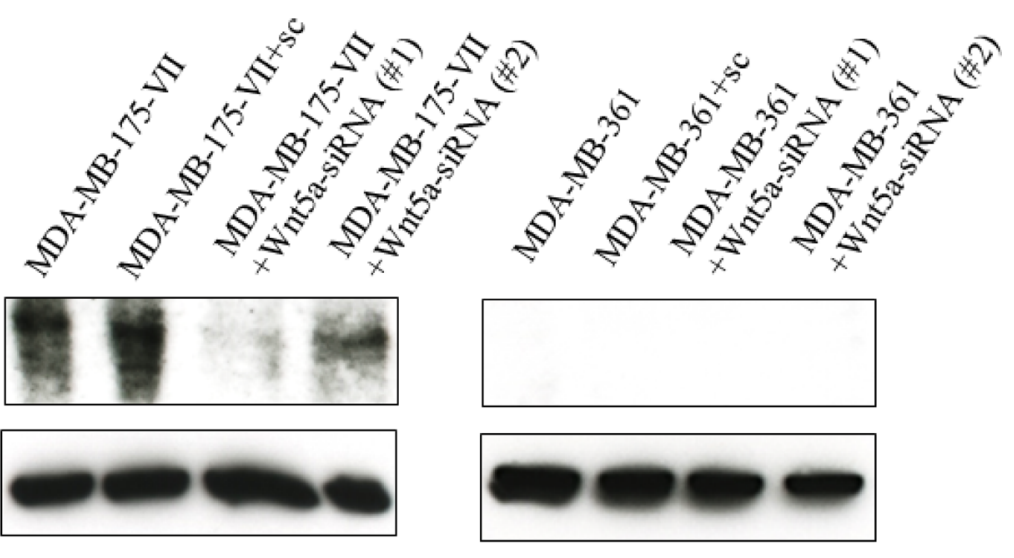

B
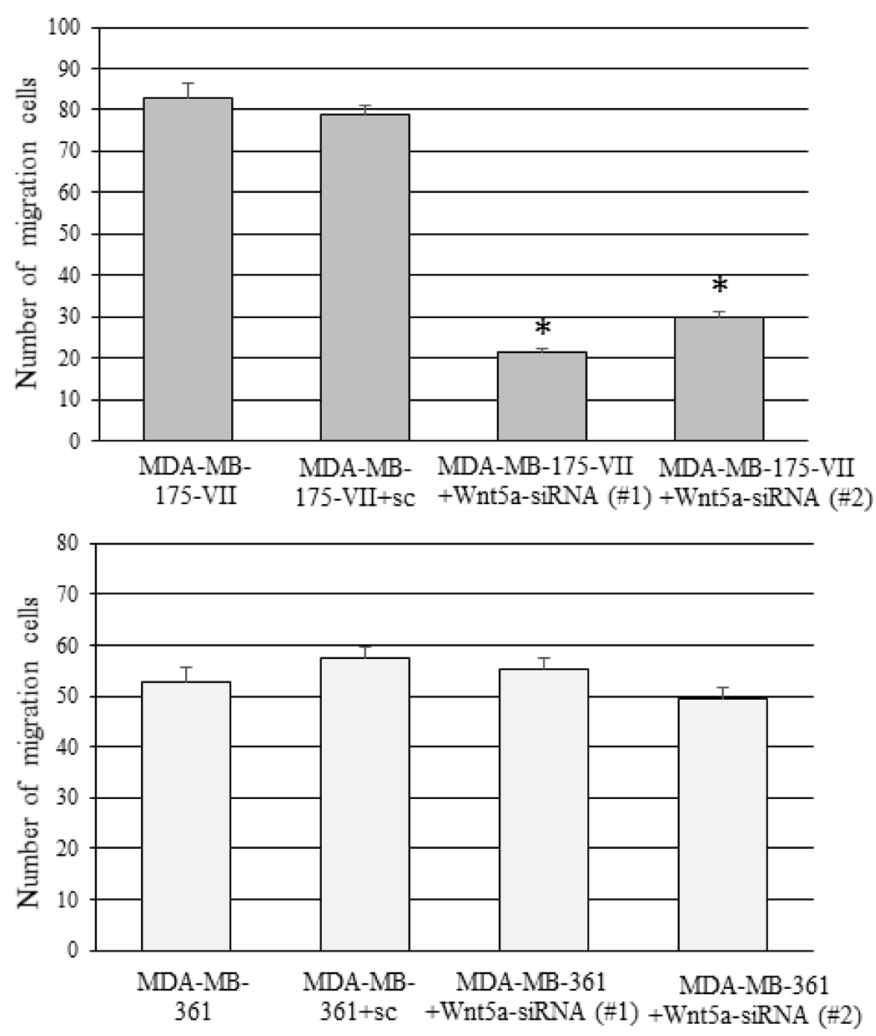

Figure 4: Knockdown of Wnt5a decreases the migratory capacity of MDA-MB-175-VII cells. (A) The Wnt5a expression of MDA-MB-175-VII and MDA-MB-361 cells transfected with Wnt5a-siRNAs (Wnt5a-siRNA \#1 and \#2) and sc. (B) The migratory capacity of MDA-MB-175-VII and MDA-MB-361 cells transfected with Wnt5a-siRNAs and sc. Data are presented as the mean \pm SE of three migration assays. Data were evaluated using the Mann-Whitney $U$-test. 
Table 3: Genes up-regulated by expression of Wnt5a

\begin{tabular}{cclccc}
\hline & & & \multicolumn{3}{c}{$\begin{array}{c}\text { Fold changes } \\
\text { (ratio MCF7 Wnt5a/MCF7 control) }\end{array}$} \\
\hline No. & Gene symbol & \multicolumn{1}{c}{ Gene name } & $\begin{array}{c}\text { MCF7 } \\
\text { Wnt5a (1) }\end{array}$ & $\begin{array}{c}\text { MCF7 } \\
\text { Wnt5a (2) }\end{array}$ & $\begin{array}{c}\text { MCF7 Wnt5a (3) } \\
\text { Wnd }\end{array}$ \\
\hline 1 & ID1 & Inhibitor of DNA binding 1 & 3.94 & 2.08 & 2.28 \\
2 & MAOA & Amine oxidase A & 2.85 & 2.26 & 4.85 \\
3 & ALCAM & Activated leukocyte cell adhesion molecule & 3.25 & 2.81 & 3.07 \\
4 & AKR1C3 & Aldo-keto reductase family 1 member C3 & 3.18 & 2.18 & 5.17 \\
5 & MARCKS & Myristoylated alanine-rich C-kinase substrate & 5.93 & 2.23 & 10.37 \\
6 & CALML5 & Calmodulin-like protein 5 & 2.87 & 2.53 & 4.10 \\
7 & NOL11 & Nucleolar protein 11 & 2.85 & 2.67 & 2.63 \\
8 & STX12 & Syntaxin-12 & 3.70 & 3.84 & 5.08 \\
9 & CCDC104 & Coiled-coil domain-containing protein 104 & 2.91 & 2.79 & 3.91 \\
10 & ITM2B & Integral membrane protein 2B & 3.13 & 3.04 & 3.18 \\
11 & CHMP4A & Charged multivesicular body protein 4a & 3.63 & 2.96 & 3.03 \\
12 & D2HGDH & D-2-hydroxyglutarate dehydrogenase & 3.23 & 3.20 & 3.76 \\
13 & CRK & Adapter molecule crk & 3.00 & 3.15 & 3.00 \\
14 & DIABLO & Direct IAP binding protein with low pI & 3.84 & 3.15 & 4.04 \\
15 & ZNF446 & Zinc finger protein 446 & 2.03 & 2.05 & 2.15 \\
\hline
\end{tabular}

Hiroshima University Hospital in Japan were sequentially enrolled in this study. Patients treated with neoadjuvant chemotherapy were excluded. This study was approved by the institutional review board of Hiroshima University Hospital (No. 926), and all participants provided written informed consent for the use of their tissue specimens. Each participant's medical information was obtained from medical records.

\section{Immunohistochemistry}

Immunohistochemistry was performed as previously described [22]. Antigen retrieval was made using proteinase treatment (Proteinase K S3004; Dako, Carpinteria, California, USA) for $2 \mathrm{~min}$ and $30 \mathrm{~s}$ in room air. The sections were reacted with the polyclonal antiWnt5a antibody, a kind gift from Prof. Akira Kikuchi (Department of Molecular Biology and Medicine, Osaka University, Suita, Japan) at $4^{\circ} \mathrm{C}$ overnight. Activated leukocyte cell adhesion molecule (ALCAM) was stained with anti-ALCAM antibody (clone MOG/07; Leica Biosystems, Wetzlar, Hesse, Germany). Antigen retrieval was made using heat-treatment for $20 \mathrm{~min}$ at $98^{\circ} \mathrm{C}$ in citrate buffer ( $\mathrm{pH}$ 6.0). Wnt5a and ALCAM expression were scored as $0,1+, 2+$, or $3+$, taking into consideration staining proportion and intensity. The Wnt5a and ALCAM scores were assessed as follows: 0 , no staining or staining in $\leq 10 \%$ of invasive tumor cells; $1+$, weak staining in
$>10 \%$ of invasive tumor cells; $2+$, moderate staining in $>10 \%$ of invasive tumor cells or strong staining in $>10 \%$ and $\leq 30 \%$ of invasive tumor cells; $3+$, strong staining in $>30 \%$ of invasive tumor cells. A score of $3+$ was defined as positive for both Wnt5a and ALCAM. The scoring was independently conducted by two investigators who had no knowledge of the patients' clinical data. An automatic staining machine was used for immunohistochemical staining of the following biomarkers: ER, PgR (SP-1, monoclonal antibody; Ventana Medical Systems, Tucson, Arizona, USA), HER2 (polyclonal antibody; Dako), and Ki-67 (30-9, monoclonal antibody; Ventana Medical Systems). A proportion of nuclear staining of ER and PgR $\geq 1 \%$ was considered positive expression. HER2 expression was evaluated accordingly as previously described [29].

\section{Cell culture and transfection}

MCF-7 cells were obtained from American Type Culture Collection (Manassas, Virginia, USA) and were grown in RPMI-1640 medium supplemented with $10 \%$ fetal bovine serum. MCF-7 cells were transiently transfected with pPGK-neo/Wnt5a using Lipofectamine LTX with PLUS reagent (Life Technologies, Carlsbad, California, USA). pPGK-neo/Wnt5a was a kind gift from Prof. Akira Kikuchi. MCF-7 cells stably expressing mouse Wnt5a (MCF-7/Wnt5a) were generated by selection with $200 \mu \mathrm{g} / \mathrm{mL}$ G418. 
Table 4: Relationship between Wnt5a expression and ALCAM expression in ER-positive breast cancer

\begin{tabular}{lccc}
\hline & \multicolumn{3}{c}{ ALCAM } \\
\cline { 2 - 4 } Total $(\boldsymbol{N}=\mathbf{1 5 3})$ & Negative $(\boldsymbol{N}=\mathbf{8 3})$ & Positive $(\boldsymbol{N}=\mathbf{7 0})$ & $\boldsymbol{P}$ value \\
\hline Wnt5a & $62(73 \%)$ & $23(27 \%)$ & $47(69 \%)$ \\
Negative $(N=85)$ & $21(31 \%)$ & 40.001 \\
Positive $(N=68)$ & & & \\
\hline
\end{tabular}

kappa coefficient $\kappa=0.444$. 95\% CI.: [0.3013, 0.5869].

We sough breast cancer cells expressing ER, Wnt5a, and ALCAM endogenously to address the mechanism by which ALCAM mediates the ability of Wnt5a to promote migration of ER-positive breast cancer cells. MDAMB-175-VII, MDA-MB-361, ZR-75-30, ZR-75-1, and CAMA-1 cells were obtained from American Type Culture Collection. MDA-MB-175-VII, MDA-MB-361, and CAMA-1 cells were grown in Leibovitz's L-15 medium supplemented with $10 \%$ fetal bovine serum, Leibovitz's L-15 medium supplemented with 20\% fetal bovine serum, and Eagle's minimum essential medium supplemented with $10 \%$ fetal bovine serum, respectively. ZR-75-30 and ZR-75-1 cells were grown in RPMI-1640 medium supplemented with $10 \%$ fetal bovine serum.

\section{SiRNA}

In analyses with small interfering RNAs (siRNAs) for Wnt5a, the human Wnt-5a mRNA target sequences, 5'-TATCAATTCCGACATCGAATT-3' and 5'-UAUCAAUUCCGACAUCGAATT-3' were used (Life Technologies). For ALCAM, the human ALCAM mRNA

A
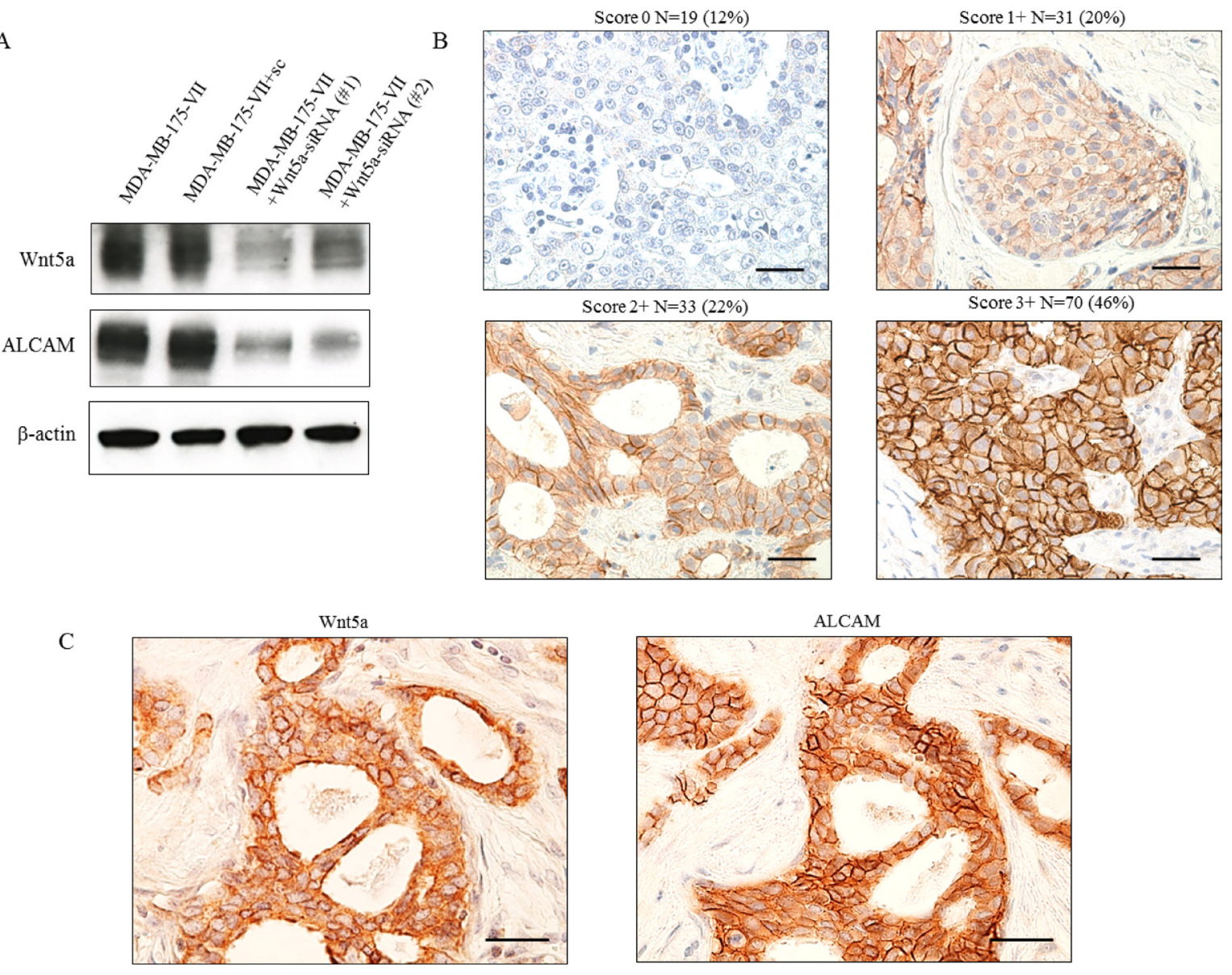

Figure 5: Co-expression of Wnt5a and ALCAM in ER-positive breast cancer tissue. (A) ALCAM expression was reduced by knockdown of Wnt5a. (B) Evaluation of ALCAM expression was scored as 0, 1+, 2+, or 3+. Scoring of ALCAM was performed with the same evaluation as Wnt5a. (C) Co-expression of Wnt5a and ALCAM in breast cancer tissue. 
target sequences, 5'-GCAUAUGGAGAUACCAUUATT3' and 5'-GGACCUCGGUAAUAUGGAATT-3' were used (Life Technologies). To transfect siRNA into MCF7 cells expressing Wnt5a, trypsinized MCF-7 cells were suspended in Opti-MEM (Life Technologies) at $10^{6}$ cells per $100 \mu \mathrm{L}$, siRNA was added (90 pmol), and cells were mixed with RNAi max (Life Technologies) in Opti-MEM.

\section{Cell migration assay}

To assess cell migration activity, a two-chamber transmigration assay was performed as previously described [36, 37]. MCF-7/Wnt5a cells $\left(5.0 \times 10^{4}\right.$ cells $)$, $5.0 \times 10^{4} \mathrm{MCF}-7 / \mathrm{Wnt} 5 \mathrm{a}$ cells + scramble $(\mathrm{sc}), 5.0 \times$
$10^{4} \mathrm{MCF}-7 / \mathrm{Wnt} 5 \mathrm{a}+\mathrm{Wnt5a}$-siRNA, $5.0 \times 10^{4} \mathrm{MCF}-7 /$ Wnt5a + ALCAM-siRNA, and $5.0 \times 10^{4} \mathrm{MCF}-7$ cells with $500 \mu \mathrm{L}$ RPMI-1640 medium were seeded into the upper chamber, and $750 \mu \mathrm{L}$ NIH3T3-conditioned medium and $750 \mu \mathrm{L}$ RPMI-1640 medium (10\% FBS) was added to the lower chamber of a 12-well Multi Well Plate (BD Biosciences, San Jose, California, USA). Cells were incubated for $12 \mathrm{~h}$ at $37^{\circ} \mathrm{C}$. The migrated cells on the underside of the upper chamber were stained with phalloidin (P1951; Sigma-Aldrich, St. Louis, Missouri, USA) and counted per 10 high-power fields $(\times 200)$ using a fluorescence microscope (FV1000; Olympus, Tokyo, Japan). These experiments were performed at least three times in each cell line.

A

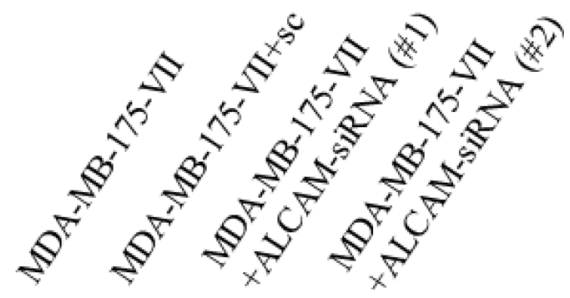

Wnt5a

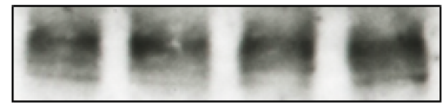

ALCAM

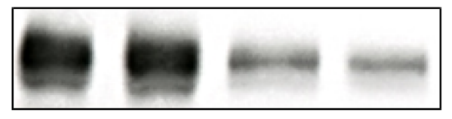

$\beta$-actin

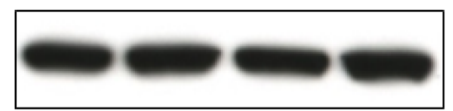

B

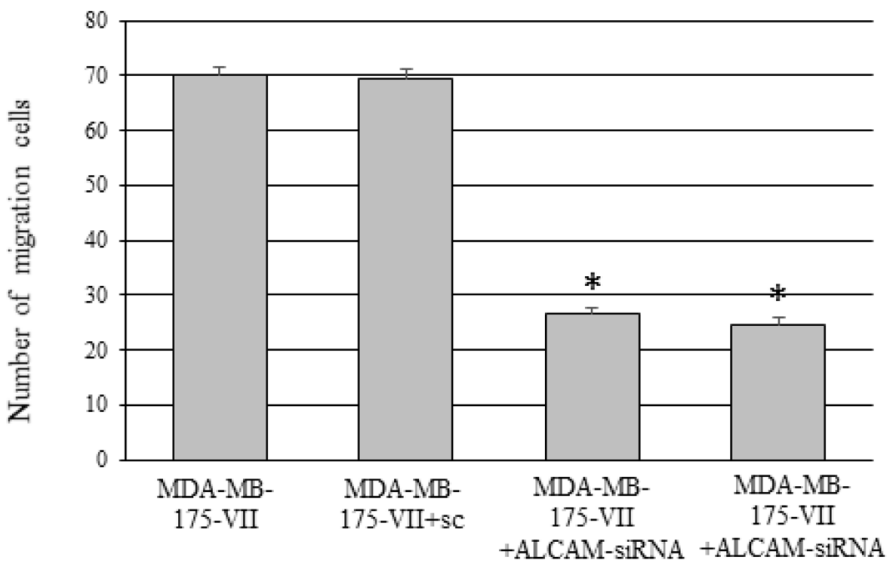

(\#1)

$(\# 2)$

Figure 6: Knockdown of ALCAM decreases the Wnt5a-induced increase in migratory capacity. (A) Wnt5a and ALCAM expression of MDA-MB-175-VII cells transfected with ALCAM-siRNAs (ALCAM-siRNA\#1 and \#2) and sc. (B) The migratory capacity of MDA-MB-175-VII cells transfected with ALCAM-siRNAs and sc. Data are presented as the mean \pm SE of three migration assays. Data were evaluated using the Mann-Whitney $U$-test. 


\section{Cell proliferation assay}

The control MCF-7 and MCF-7/Wnt5a cells were seeded at densities of $1 \times 10^{5} / \mathrm{mL}$ as previously described [8]. Cells were enumerated on the incubated days.

\section{Gene microarray analysis}

For the Oligo DNA microarray analysis, cells were washed with phosphate-buffered saline once and collected with Buffer RLT (Qiagen, Hilden, North Rhine-Westphalia, Germany). Total RNA was extracted from cells using the RNeasy mini kit (Qiagen). The 3D-Gene Human Oligo chip $25 \mathrm{k}$ (Toray Industries, Tokyo, Japan) was used. For efficient hybridization, this microarray has 3-dimensions constructed with a well as the space between the probes and cylinder-stems, with 70-mer oligonucleotide probes on the top. Total RNA was labeled with Cy5 using the Amino Allyl MessageAMP II aRNA Amplification Kit (Applied Biosystems, Foster City, California, USA). The Cy5labeled aRNA pools were placed in hybridization buffer and allowed to hybridize for $16 \mathrm{~h}$ using the supplier's protocols (www.3d-gene.com). The hybridization signals were obtained using the 3D-Gene Scanner (Toray Industries) and processed by 3D-Gene Extraction (Toray Industries). Detected signals for each gene were normalized using the global normalization method, with the median of the detected signal intensity adjusted to 25 .

\section{Materials and chemicals}

Anti-Wnt5a/b antibody was purchased from Cell Signaling Technology (Beverly, Massachusetts, USA). Anti- $\beta$-catenin and anti-ALCAM antibodies were purchased from BD Biosciences and Leica Biosystems, respectively. Anti-ER and anti-ErbB 2 antibodies were purchased from Abcam. Anti- $\beta$-actin and anti-cyclin D1 antibodies were purchased from Sigma-Aldrich.

\section{Statistical analysis}

The correlation between immunohistochemical determination of Wnt5a expression and clinicopathologic factors in tumor tissues was analyzed using the Chisquare test. The correlation between Wnt5a and ALCAM expression was analyzed by kappa coefficient. The MannWhitney $U$-test was used to compare cell migration. $P$ values $<0.05$ were considered statistically significant. All calculations were performed using SPSS version 20.

\section{Abbreviations}

JNK: c-Jun N-terminal kinase; PIK3CA: phosphatidylinositol-4,5-bisphosphate 3-kinase catalytic subunit alpha; FAK: focal adhesion kinase; Rac: RASrelated C3 botulinus toxin substrate; Ror: receptor tyrosine kinase-like orphan receptor; MCF-7: Michigan Cancer Foundation7; RPMI: Roswell Park Memorial Institute medium; pPGK-neo: plasmid phosphoglycerate kinase 1 promoter-neomycin; PI3K: phosphatidylinositol 3-kinase; mTOR: mammalian target of rapamycin; SE: standard error.

\section{Author contributions}

TK conceived and directed this study. He also conducted transfection, Western blotting, and drafted the manuscript. YK conducted immunohistochemistry, cell culture, Western blotting, statistical analysis, and cell migration assay and drafted the manuscript. AA contributed to data collection and performed the manual review. HY and $\mathrm{AK}$ helped to interpret the data and discussed the manuscript. KA evaluated immunohistochemistry and advised on data analysis. MO advised on study design. All authors performed critical review of the manuscript. All authors read and approved the final manuscript.

\section{ACKNOWLEDGMENTS}

We thank Ze'ev Ronai (Sanford Burnham Prebys Medical Discovery Institute, La Jolla, California, USA) for his critical review of this manuscript.

\section{CONFLICTS OF INTEREST}

No potential conflicts of interest were disclosed.

\section{FUNDING}

This study was supported by Grant-in-Aid for Scientific Research C, Japan Society for the Promotion of Science, JSPS KAKENHI Grant Number JP26461947 (to TK), 26861049 (to KK), and 17K16509 (to AA).

\section{REFERENCES}

1. Nusse R. Wnt signaling in disease and in development. Cell Res. 2005; 15:28-32.

2. Kikuchi A, Yamamoto H, Sato A. Selective activation mechanisms of Wnt signaling pathways. Trends Cell Biol. 2009; 19:119-29.

3. Polakis P. The many ways of Wnt in cancer. Curr Opin Genet Dev. 2007; 17:45-51.

4. Kikuchi A, Yamamoto H, Sato A, Matsumoto S. Wnt5a: its signalling, functions and implication in diseases. Acta Physiol. 2012; 204:17-33.

5. Bittner M, Meltzer P, Chen Y, Jiang Y, Seftor E, Hendrix M, Radmacher M, Simon R, Yakhini Z, Ben-Dor A, Sampas N, Dougherty E, Wang E, et al. Molecular classification of cutaneous malignant melanoma by gene expression profiling. Nature. 2000; 406:536-40. 
6. Weeraratna AT, Jiang Y, Hostetter G, Rosenblatt K, Duray P, Bittner M, Trent JM. Wnt5a signaling directly affects cell motility and invasion of metastatic melanoma. Cancer Cell. 2002; 1:279-88.

7. Kurayoshi M, Oue N, Yamamoto H, Kishida M, Inoue A, Asahara T, Yasui W, Kikuchi A. Expression of Wnt-5a is correlated with aggressiveness of gastric cancer by stimulating cell migration and invasion. Cancer Res. 2006; 66:10439-48.

8. Hanaki H, Yamamoto H, Sakane H, Matsumoto S, Ohdan H, Sato A, Kikuchi A. An anti-Wnt5a antibody suppresses metastasis of gastric cancer cells in vivo by inhibiting receptor-mediated endocytosis. Mol Cancer Ther. 2012; 11:298-307.

9. Yamamoto H, Kitadai Y, Yamamoto H, Oue N, Ohdan H, Yasui W, Kikuchi A. Laminin $\gamma 2$ mediates Wnt5a-induced invasion of gastric cancer cells. Gastroenterology. 2009; 137:242-52.

10. Yamamoto H, Oue N, Sato A, Hasegawa Y, Yamamoto H, Matsubara A, Yasui W, Kikuchi A. Wnt5a signaling is involved in the aggressiveness of prostate cancer and expression of metalloproteinase. Oncogene. 2010; 29:2036-46.

11. Huang CL, Liu D, Nakano J, Ishikawa A, Kontani K, Yokomise H, Ueno M. Wnt5a expression is associated with the tumor proliferation and the stromal vascular endothelial growth factor-an expression in non-small cell lung cancer. J Clin Oncol. 2005; 23:8765-73.

12. Ripka S, König A, Buchholz M, Wagner M, Sipos B, Klöppel G, Downward J, Gress T, Michl P. WNT5A-target of CUTL1 and potent modulator of tumor cell migration and invasion in pancreatic cancer. Carcinogenesis. 2007; 28:1178-87.

13. Dejmek J, Dejmek A, Säfholm A, Sjölander A, Andersson T. Wnt-5a protein expression in primary dukes B colon cancers identifies a subgroup of patients with good prognosis. Cancer Res. 2005; 65:9142-6.

14. Kremenevskaja N, von Wasielewski R, Rao AS, Schöfl C, Andersson T, Brabant G. Wnt-5a has tumor suppressor activity in thyroid carcinoma. Oncogene. 2005; 24:2144-54.

15. Lju XH, Pan MH, Lu ZF, Wu B, Rao Q, Zhou ZY, Zhou XJ. Expression of Wnt-5a and its clinicopathological significance in hepatocellular carcinoma. Dig Liver Dis. 2008; 40:560-7.

16. Liang H, Chen Q, Coles AH, Anderson SJ, Pihan G, Bradley A, Gerstein R, Jurecic R, Jones SN. Wnt5a inhibits $\mathrm{B}$ cell proliferation and functions as a tumor suppressor in hematopoietic tissue. Cancer Cell. 2003; 4:349-60.

17. Jönsson M, Dejmek J, Bendahl PO, Andersson T. Loss of Wnt-5a protein is associated with early relapse in invasive ductal breast carcinomas. Cancer Res. 2002; 62:409-16.

18. Sand-Dejmek J, Ehrnström R, Berglund P, Andersson T, Ryden L. The prognostic significance of Wnt-5a expression in primary breast cancer is extended to premenopausal women. PLoS One. 2013; 8:e70890.
19. Onitilo AA, Engel JM, Geenlee RT, Mukesh BN. Breast cancer subtypes based on ER/PR and Her2 expression: comparison of clinicopathologic features and survival. Clin Med Res. 2009; 7:4-13.

20. Carey LA, Perou CM, Livasy CA, Dressler LG, Cowan D, Conway K, Karaca G, Troester MA, Tse CK, Edmiston S, Deming SL, Geradts J, Cheang MC, et al. Race, breast cancer subtypes, and survival in the Carolina breast cancer study. JAMA. 2006; 295:2492-502.

21. Cizkova M, Cizeron-Clairac G, Vacher S, Susini A, Andrieu C, Lidereau R, Bièche I. Gene expression profiling reveals new aspects of PIK3CA mutation in ERalpha-positive breast cancer: major implication of the Wnt signaling pathway. PLoS One. 2010; 5:e15647.

22. Dejmek J, Leandersson K, Manjer J, Bjartell A, Emdin SO, Vogel WF, Landberg G, Andersson T. Expression and signaling activity of Wnt-5a/discoidin domain receptor-1 and Syk plays distinct but decisive roles in breast cancer patient survival. Clin Cancer Res. 2005; 11:520-8.

23. Klemm F, Bleckmann A, Siam L, Chuang HN, Rietkötter E, Behme D, Schulz M, Schaffrinski M, Schindler S, Trümper L, Kramer F, Beissbarth T, Stadelmann C, et al. $\beta$-cateninindependent WNT signaling in basal-like breast cancer and brain metastasis. Carcinogenesis. 2011; 32:434-42.

24. Pukrop T, Klemm F, Hagemann T, Gradl D, Schulz M, Siemes S, Trümper L, Binder C. Wnt 5a signaling is critical for macrophage-induced invasion of breast cancer cell lines. Proc Natl Acad Sci USA. 2006; 103:5454-9.

25. Zhu Y, Tian Y, Du J, Hu Z, Yang L, Liu J, Gu L. Dvl2dependent activation of Daam1 and RhoA regulates Wnt5ainduced breast cancer cell migration. PLoS One. 2012; 7:e37823.

26. Hein S, Müller V, Köhler N, Wikman H, Krenkel S, Streichert T, Schweizer M, Riethdorf S, Assmann V, Ihnen M, Beck K, Issa R, Jänicke F, et al. Biologic role activated leukocyte cell adhesion molecule overexpression in breast cancer cell lines and clinical tumor tissue. Breast Cancer Res Treat. 2011; 129:347-60.

27. Jezierska A, Matysiak W, Motyl T. ALCAM/CD166 protects breast cancer cells against apoptosis and autophagy. Med Sci Monit. 2006; 12:BR263-73.

28. van Kilsdonk JW, Wilting RH, Bergers M, van Muijen GN, Schalkwijk J, van Kempen LC, Swart GW. Attenuation of melanoma invasion by a secreted variant of activated leukocyte cell adhesion molecule. Cancer Res. 2008; 68:3671-9.

29. Wolff AC, Hammond ME, Schwartz JN, Hagerty KL, Allred DC, Cote RJ, Dowsett M, Fitzgibbons PL, Hanna WM, Langer A, McShane LM, Paik S, Pegram MD, et al. American Society of Clinical Oncology/College of American Pathologists. American society of clinical oncology/college of american pathologists guideline recommendations for human epidermal growth factor receptor 2 testing in breast cancer. Arch Pathol Lab Med. 2007; 131:18-43. 
30. Piao D, Jiang T, Liu G, Wang B, Xu J, Zhu A. Clinical implications of activated leukocyte cell adhesion molecule expression in breast cancer. Mol Biol Rep. 2012; 39:661-8.

31. Ihnen M, Müller V, Wirtz RM, Schröder C, Krenkel S, Witzel I, Lisboa BW, Jänicke F, Milde-Langosch K. Predictive impact of activated leukocyte cell adhesion molecule (ALCAM/CD166) in breast cancer. Breast Cancer Res Treat. 2008; 112:419-27.

32. Cizelsky W, Tata A, Kühl M, Kühl SJ. The Wnt/JNK signaling target gene alcam is required for embryonic kidney development. Development. 2014; 141:2064-74.

33. Yang SX, Polley E, Lipkowitz S. New insights on PI3K/ AKT pathway alterations and clinical outcomes in breast cancer. Cancer Treat Rev. 2016; 45:87-96.

34. Braga S. Resistance to targeted therapies in breast cancer. Methods Mol Biol. 2016; 1395:105-36.
35. Maeda K, Kobayashi Y, Udagawa N, Uehara S, Ishihara A, Mizoguchi T, Kikuchi Y, Takada I, Kato S, Kani S, Nishita M, Marumo K, Martin TJ, et al. Wnt5a-Ror2 signaling between osteoblast-lineage cells and osteoclast precursors enhances osteoclastogenesis. Nat Med. 2012; 18:405-12.

36. Ito M, Hagiyama M, Mimae $\mathrm{T}$, Inoue $\mathrm{T}$, Kato $\mathrm{T}$, Yoneshige A, Nakanishi J, Kondo T, Okada M, Ito A. $\alpha$-Parvin, a pseudopodial constituent, promotes cell motility and is associated with lymph node metastasis of lobular breast carcinoma. Breast Cancer Res Treat. 2014; 144:59-69.

37. Zhang G, Brewster A, Guan B, Fan Z, Brown PH, Xu XC. Tumor-suppressor activity of RRIG1 in breast cancer. BMC Cancer. 2011; 11:32. 